\title{
Variations du rendement fromager en pâte molle. Relations avec la composition du lait et les paramètres de la coagulation
}

\author{
O Colin, F Laurent, B Vignon
}

INRA-ENSAIA, laboratoire des sciences et techniques des productions animales 2, av de la forêt de Haye, BP 172, 54505 Vandceuvre-lès-Nancy Cedex, France

(Reçu le 31 mai 1991; accepté le 10 février 1992)

\begin{abstract}
Résumé - Des transformations fromagères de type pâte molle ont permis d'étudier l'influence des variations de la teneur en protéines (TP) et de la nature de ces protéines sur le rendement fromager. Ces relations ont été comparées à celles obtenues avec les paramètres de la coagulation mesurés au Formagraph. Les fabrications pilotes sont réalisées avec des laits de mélange à 4 niveaux différents de $\operatorname{TP}(29,4 ; 30,1 ; 30,8 ; 32,2 \mathrm{~g} / \mathrm{l}) .4$ répétitions effectuées à 1 semaine d'intervalle portent sur $40 \mathrm{~kg}$ de lait réfrigéré $24 \mathrm{~h}$ et écrémé partiellement (taux butyreux $=26,6 \mathrm{~g} / \mathrm{l}$ ). Le pourcentage de caséines dans les protéines totales et les pourcentages des différentes caséines $\left(\alpha_{s}, \beta, \gamma+\kappa\right)$ dans l'ensemble des caséines ne varient pas significativement avec le niveau de TP. Le taux butyreux et la teneur en protéines permettent d'expliquer $77 \%$ des variations du rendement fromager frais mesuré 1 j après transformation, alors que le temps d'obtention de la fermeté standard $\left(K_{20}\right)$ explique $74 \%$ des variations. La meilleure prédiction du rendement fromager frais est établie à partir du $K_{20}$ et de la teneur en protéines $\left(r^{2}=0,86\right)$. Le rendement ajusté en matière sèche (MS) ou le rendement exprimé en MS (rendement frais $x$ teneur en MS des fromages) sont expliqués au mieux par la seule teneur en protéines ( $r^{2}=0,66$ et 0,71 respectivement); les paramètres de la coagulation ne sont pas corrélés significativement au rendement ajusté en MS ou au rendement sec. La prise en compte de la teneur en caséines totales au lieu de la teneur en protéines n'améliore pas la prédiction du rendement frais mais l'augmente pour le rendement ajusté en MS ou le rendement sec. Parmi les différentes caséines, seule la teneur en caséine $\beta$ du lait explique une part importante des variations du rendement frais $\left(r^{2}=0.63\right)$.
\end{abstract}

rendement / fromage pâte molle / protéines / paramètres de coagulation / lait de vache

Summary - Relations between soft cheese yield variations and milk composition or coagulation parameter variations. Laboratory-scale soft cheese making was carried out to quantify the influence of true protein content variations and of the different proteins on cheese yield. These relations have been compared with the predictions obtained when using coagulation parameters measured with a Formagraph. Laboratory-scale cheese making was carried out with bulk milks with 4 different levels of true protein content $(29.4 ; 30.1 ; 30.8 ; 32.2 \mathrm{~g} / \mathrm{l})$. Four tests, carried out every 7 days, were made with $40 \mathrm{~kg}$ of cooled and fat standardized milk (fat $=26.6 \mathrm{~g} / \mathrm{l}$ ). The percentage of caseins in total proteins and the percentage of different caseins $\left(\alpha_{s}, \beta, \gamma+\kappa\right)$ in total casein did not differ significantly with level of true protein content. Seventy-seven percent of the variations in gross cheese yield, when measured 1 day after clotting, could be explained from fat and true protein contents while $74 \%$ of the variations could be explained from rate of firming $\left(\mathrm{K}_{20}\right)$. The best prediction 
of gross cheese yield was established by using $\mathrm{K}_{20}$ and true protein content $\left(\mathrm{r}^{2}=0.86\right)$. The best prediction of cheese yield adjusted on dry matter and of dry matter yield (gross cheese yield $x$ cheese dry matter) was established by using only true protein content $\left(r^{2}=0.66\right.$ and 0.71 respectively). Coagulation parameters were not significantly correlated with cheese yield adjusted on the dry matter or with dry matter yield. The use of casein content instead of true protein content did not improve the prediction of gross cheese yield but increased it for dry matter adjusted cheese yield or for dry matter yield. Among the different caseins, only $\beta$ casein content explained a large part of the variation in gross cheese yield $\left(r^{2}=0.63\right)$.

\section{yield / soft cheese / true protein / coagulation parameters / bovine milk}

\section{INTRODUCTION}

De nombreux tests d'estimation de l'aptitude fromagère du lait s'attachent à caractériser les phases de coagulation et d'égouttage en définissant les vitesses de coagulation et de raffermissement du coagulum. Bien que les caractéristiques rhéologiques du coagulum aient un rôle déterminant sur les pertes de matière dans le sérum et les caractéristiques finales du fromage (Kerjean, 1984), jusqu'à ce jour aucune des mesures de la coagulation des laits ou de la fermeté des gels ne permet d'avoir une estimation très précise du rendement fromager obtenu en fin de fabrication (Aleandri et al, 1989; Hurtaud et al, 1991).

II est toujours important de quantifier l'influence directe des paramètres de composition physico-chimique du lait sur le rendement fromager. Les équations de prédiction du rendement à partir de la composition du lait ont surtout été établies en fabrication de pâte pressée (Mocquot et al, 1963; Maubois et al, 1970; Banks et al, 1984; Gilles et Lawrence, 1985; Aleandri et al, 1989; Emmons et al, 1990; Garel et Coulon, 1990; Hardy, 1990). Quelques auteurs ont également cherché à expliquer les variations de rendement en fabrication de pâte molle (Maubois et al, 1970). Ces travaux montrent que l'importance des différents constituants du lait dans la prédiction du rendement varie fortement, notamment en fonction des technologies utilisées.
L'objectif de cette étude est de 2 ordres: - d'une part, quantifier l'influence de la teneur en protéines du lait sur le rendement fromager en fabrication de type pâte molle et préciser le poids relatif des différentes protéines du lait, particulièrement des caséines, dans la prédiction du rendement;

- d'autre part, expliquer les variations du rendement fromager à partir des variations des paramètres de la coagulation afin de préciser leur validité comme prédicteurs du rendement et comparer les prédictions établies à partir des seuls critères de composition physico-chimique du lait avec celles utilisant les paramètres de la coagulation.

\section{MATÉRIEL ET MÉTHODES}

\section{Protocole expérimental}

Des fabrications pilotes sont réalisées avec des laits de mélange à 4 teneurs différentes en protéines, choisies de manière à obtenir une gamme de TP la plus large possible.

Pour simuler au mieux les conditions industrielles et atténuer les perturbations liées à un effet troupeau, chaque lait mis en fabrication est constitué par une fraction du mélange des laits de 2 traites consécutives de 3 troupeaux, réfrigérés $24 \mathrm{~h}$ en tank à la ferme. Les fabrications, de type pâte molle, portent sur $40 \mathrm{~kg}$ de lait, standardisé à environ $26 \%$ de matière grasse (MG).

L'expérimentation se déroule en période hivernale, caractérisée par une alimentation stable et une faible fréquence de vêlages. 
Quatre fabrications successives pour chaque niveau de $T P$ sont réalisées à 1 semaine d'intervalle au cours de cette période.

\section{Analyses et mesures}

Les mesures suivantes sont réalisées sur chaque lait mis en fabrication :

- teneur en matière sèche (MS) par dessication à $103^{\circ} \mathrm{C} \pm 2^{\circ} \mathrm{C}$ pendant $24 \mathrm{~h}$,

- taux butyreux (analyseur Irma),

- teneurs en azote total $\left(N_{\text {total }}\right)$, azote non protéique (ANP) après précipitation des protéines à l'acide trichloroacétique $(12 \%$ de la solution finale), azote soluble $\left(N_{\text {sol }}\right)$ après précipitation des caséines à l'acide acétique $(1 \mathrm{~N})$ par ajustement du $\mathrm{pH}$ à 4,6 (méthode de Kjeldahl, dosage automatique sur Vapodest 6).

- nature et pourcentage des caséines et protéines du lactosérum par chromatographie FPLC et HPLC respectivement (méthode d'Andrews et al, 1985),

- teneurs en $\mathrm{Ca}, \mathrm{Na}$ et $\mathrm{Mg}$ totaux par spectrophotométrie d'absorption atomique (flamme : air-acétylène, longueur d'onde : 423,589 et 285 $\mathrm{nm}$, largeur de fente : $0,7,0,2$ et $0,7 \mathrm{~nm}$ respectivement). La prise d'essai est constitué d'1 ml de solution de cendres (obtenues par calcination au four à moufle, $550^{\circ} \mathrm{C}$ durant 5 h) diluée 200 fois par ajout de $20 \mathrm{ml}$ de chlorure de lanthane complèté avec de l'eau distillée,

- teneur en $\mathrm{P}$ total par colorimétrie (spectrophotomètre à $660 \mathrm{~nm}$ ). La prise d'essai est constituée d'1 $\mathrm{ml}$ de solution de cendres diluée 25 fois par ajout de $0,4 \mathrm{ml}$ d'acide ascorbique $1 \%$ et 0,8 ml de molybdate d'ammonium 2,5\% complété avec de l'eau distillée.

- taille micellaire (granulozétamètre Malvern),

$-\mathrm{pH}$,

- temps de coagulation $(R)$ et temps d'obtention de la fermeté standard $\left(K_{20}\right)$ (Formagraph). Les échantillons, constitués par $10 \mathrm{ml}$ de lait écrémé, sont portés à la température de $35^{\circ} \mathrm{C} \quad(30-45$ min) puis emprésurés par $200 \mu l$ d'une solution de présure à $7,8 \mathrm{mg}$ de chymosine active par $\mathrm{l}$.

Les teneurs en protéines (TP) et en caséines ( $T$ Cas), exprimées en $\mathrm{g}$ de protéines par I de lait, sont obtenues à partir des teneurs en azote protéique $\left(N_{\text {total }}-A N P\right)$ et en azote caséique $\left(N_{\text {total }}-N_{\text {sol }}\right)$ par utilisation du coefficient correctif 6,38 correspondant à une teneur en azote des protéines du lait de $15,67 \%$ bien que la valeur de ce coefficient soit remise en cause par Ribadeau-Dumas et Grappin (1989).

\section{Fabrications pilotes}

Les laits sont réchauffés à $35-40^{\circ} \mathrm{C}$ pour être. standardisés en MG par écrémage total d'un volume partiel, puis ensemencés avec des levains lyophilisés acidifiants mésophiles (Eurozyme MA400) à raison de $5 \mathrm{~g}$ pour $100 \mathrm{~kg}$ de lait.

La température de la salle de transformation est maintenue à $25^{\circ} \mathrm{C}$ et l'hygrométrie à $80 \%$. Les laits sont emprésurés avec une présure titrant $520 \mathrm{mg}$ de chymosine active par litre (force $1 / 10000$ ), à raison de $25 \mathrm{ml}$ par $100 \mathrm{~kg}$ de lait et à une température de $34^{\circ} \mathrm{C}$. Le temps de floculation $\left(T_{1}\right)$ est estimé visuellement : la floculation est considérée comme ayant lieu dès la formation d'un gel.

Après 2,5 $T_{f}$ le caillé est tranché en colonnes de $2 \times 2 \mathrm{~cm}$, puis moulé à la louche $3 T_{f}$ après tranchage. On procède ensuite à 3 retournements du caillé (environ $4 \mathrm{~h}, 6 \mathrm{~h}$ et $18 \mathrm{~h}$ après le moulage). Le démoulage a lieu environ $24 \mathrm{~h}$ après le moulage. Les fromages sont alors placés en salle d'affinage.

Les paramètres de transformation fromagère et de composition des fromages mesurés sont :

- cinétique d'égouttage toutes les $30 \mathrm{~min}$ jusqu'à $6 \mathrm{~h}$ puis à $18 \mathrm{~h}$ et $24 \mathrm{~h}$;

- teneur en azote total du lactosérum (méthode de Kjeldahl) déterminée sur un échantillon représentatif du lactosérum égoutté au cours de la fabrication;

- pesée des fromages au démoulage $(J+1)$;

- détermination des teneurs en MS sur un fromage de poids moyen par fabrication à $J+1$.

La teneur du lait en matières azotées retenues dans le caillé au démoulage (MAC) est calculée à partir de la teneur en azote du lactosérum et de la quantité de lactosérum écoulé :

$$
M A C=\frac{\left(N_{t \mid} \times Q_{t t}\right)-\left(N_{\text {lact }} \times Q_{\text {lact }}\right)}{Q_{h t}} \times 6,38
$$

$N_{t /}=N$ total lait; $Q_{t t}=$ quantité de lait transformé; $N_{\text {lact }}=\mathrm{N}$ du lactoserum; $Q_{\text {lact }}=$ quantité de lactosérum.

Le rendement fromager frais correspond au poids de fromage obtenu au démoulage pour 100 $\mathrm{kg}$ de lait mis en œuvre. Le rendement fromager 
sec est défini comme le rendement frais que multiplie la teneur en MS du fromage. Le rendement ajusté en MS est calculé selon la correction proposée par Maubois et Mocquot (1967) :

Rendement ajusté en MS =

$$
\text { Rdt frais } \times \frac{M S_{f}-M S_{\text {lact }}}{M S_{\text {moy }}-M S_{\text {lact }}}
$$

$R d t_{\text {trais }}=$ rendement frais; $M S_{1}=$ matière sèche du fromage; $M S_{\text {lact }}=$ matière sèche du lactosérum; $M S_{\text {moy }}=$ matière sèche moyenne des fromages fabriqués.

\section{Traitement des données}

Une analyse de variance (procédure GLM, logiciel SAS) est réalisée pour tester la signification des différences de valeur des paramètres de composition physico-chimique, technologiques et des rendements fromagers entre chaque niveau de TP (niveau de TP: 3 degrés de libertés; erreur résiduelle : 11 degrés de liberté).

Des régressions linéaires multiples (logiciel Statitcf) sont effectuées pour expliquer les variations de rendement fromager. Elles prennent en compte successivement les teneurs en protéines (protéines totales, caséines, matières azotées coagulables) en cumul avec le taux butyreux et les paramètres de coagulation, seuls dans un premier temps puis en cumul avec les composants chimiques.

\section{RÉSULTATS}

\section{Caractéristiques des laits transformés}

Les laits mis en fabrication sont issus de troupeaux caractérisés par un niveau de production se situant entre 5200 et 8000 $\mathrm{kg} . \mathrm{an}^{-1}$ par vache et des vêlages d'automne-hiver $(60 \%)$. Les laits présentent de faibles numération cellulaire (193000 leucocytes $/ \mathrm{ml}$ ) et contamination bactérienne (34 000 germes $/ \mathrm{ml}$ en moyenne sur les 4 prélèvements successifs).
Après écrémage partiel, le TB des laits mis en fabrication varie entre 24,5 et $28,8 \mathrm{~g} /$ I mais le TB moyen n'est pas statistiquement différent entre les niveaux de TP (tableau I).

La teneur en protéines de ces laits varie entre 28,6 et $32,8 \mathrm{~g} / \mathrm{l}$, la teneur en caséines entre 24,3 , et $27,2 \mathrm{~g} / \mathrm{l}$ et la teneur en matières azotées du lait retenues dans le caillé au démoulage entre 22,9 et 26,0 $\mathrm{g} / \mathrm{l}$. Ces teneurs varient significativement avec le niveau de $\operatorname{TP}(P<0,01)$ (tableau I).

L'écrémage partiel des laits n'ayant pas été effectué en fonction de leur teneur en protéines, le rapport TP/TB augmente avec le niveau de $T P(P<0,05)$.

Le pourcentage de caséines dans les protéines totales, les pourcentages des différentes caséines $\left(\alpha_{s}, \beta\right.$ et $\left.\gamma+\kappa\right)$ ne varient pas significativement avec le niveau de TP. Le pourcentage des différentes protéines du lactosérum varie avec le niveau de TP mais les différences ne sont significatives que pour la $\beta$-lactoglobuline $\mathrm{A}(P<0,01)$.

Les teneurs en minéraux, le $\mathrm{pH}$ et la taille micellaire des laits ne sont pas significativement influencés par le niveau de TP (tableau I).

Le temps de coagulation $(R)$ et surtout le temps d'obtention de la fermeté standard $\left(K_{20}\right)$ ont tendance à décroître avec l'augmentation du TP mais les différences ne sont pas significatives, la variabilité pour un même niveau de TP étant importante.

\section{Caractéristiques des transformations et des fromages fabriqués}

Pour les laits à TP élevé, la quantité de lactosérum égouttée à 24 h est légèrement plus faible $(P<0,05)$ (tableau II). Les coagulums des laits à $T P$ faible ont tendance à s'égoutter plus rapidement (fig 1), la différence étant significative jusqu'à 3 h d'égouttage. 
Tableau I. Valeurs moyennes des caractéristiques physicochimiques et technologiques des laits par niveau de TP.

Mean values of physico-chemical and technological characteristics of the milks by level of true protein content.

\begin{tabular}{|c|c|c|c|c|c|}
\hline & $\begin{array}{l}\text { TP faibles } \\
\qquad n=4\end{array}$ & $\begin{array}{l}\text { TP demi- } \\
\text { faibles } \\
\mathrm{n}=3\end{array}$ & $\begin{array}{l}\text { TP demi-forts } \\
n=4\end{array}$ & $\begin{array}{l}\text { TP forts } \\
n=4\end{array}$ & $\begin{array}{c}\text { Ensemble } \\
\text { des laits } \\
\mathrm{n}=15\end{array}$ \\
\hline $\begin{array}{l}\text { Extrait sec (\%) } \\
\text { lait standardisé MG }\end{array}$ & $11,2(0,2)$ & $11,1(0,04)$ & $11,2(0,1)$ & $11,3(0,2)$ & $11,2(0,2)$ \\
\hline$T B$ initial $(g / l)^{* *}$ & $38,0(0,7)$ & $37,7(1,0)$ & $39,4(1,6)$ & $40,7(0,9)$ & $39,1(1,6)$ \\
\hline TB après écrémage partiel $(g / l)$ & $26,9(0,4)$ & $26,2(0,5)$ & $26,5(1,3)$ & $26,6(1,8)$ & $26,6(1,1)$ \\
\hline Teneur en protéines ${ }^{\star \star \star}(\mathrm{g} / \mathrm{l})$ & $29,4(0,6)$ & $30,1(0,8)$ & $30,8(0,3)$ & $32,2(0,5)$ & $30,7(1,2)$ \\
\hline$T P / T B^{* *}$ & 1,09 & 1,15 & 1,17 & 1,21 & 1,15 \\
\hline Teneur en caséines ${ }^{* \star *}(g / l)$ & $25,0(0,3)$ & $25,3(0,9)$ & $25,7(0,6)$ & $27,1(0,1)$ & $25,8(1,0)$ \\
\hline Teneur en $M A C^{* \star *}(g / l)$ & $23,7(0,6)$ & $24,0(0,8)$ & $24,6(0,2)$ & $25,9(0,2)$ & $24,6(1,0)$ \\
\hline Cas dans les protéines (\%) & $84,9(0,9)$ & $84,3(0,8)$ & $83,4(2,3)$ & $84,2(1,1)$ & $84,2(1,4)$ \\
\hline $\begin{array}{cc}\text { Caséines } & (\%) \\
\alpha_{\mathrm{s}}-\mathrm{CN} & (\%) \\
\beta-\mathrm{CN} & (\%) \\
\gamma \kappa-\mathrm{CN} & (\%)\end{array}$ & $\begin{array}{l}45,2(1,2) \\
37,4(1,1) \\
17,4(1,4)\end{array}$ & $\begin{array}{l}45,2(1,6) \\
36,7(3,0) \\
18,0(2,5)\end{array}$ & $\begin{array}{l}44,4(1,0) \\
38,0(2,2) \\
17,6(1,9)\end{array}$ & $\begin{array}{l}44,4(1,5) \\
36,9(1,8) \\
18,7(2,4)\end{array}$ & $\begin{array}{l}44,8(1,3) \\
37,0(1,9) \\
18,2(1,7)\end{array}$ \\
\hline $\begin{array}{ll}\text { Protéines du lactosérum (\%) } \\
\alpha-\mathrm{La} & (\%) \\
\beta-\mathrm{CN} & (\%) \\
\gamma \kappa-\mathrm{CN} & (\%)\end{array}$ & $\begin{array}{l}45,2(1,2) \\
37,4(1,1) \\
17,4(1,4)\end{array}$ & $\begin{array}{l}45,2(1,6) \\
36,7(3,0) \\
18,0(2,5)\end{array}$ & $\begin{array}{l}44,4(1,0) \\
38,0(2,2) \\
17,6(1,9)\end{array}$ & $\begin{array}{l}44,4(1,5) \\
36,9(1,8) \\
18,7(2,4)\end{array}$ & $\begin{array}{l}44,8(1,3) \\
37,0(1,9) \\
18,2(1,7)\end{array}$ \\
\hline $\begin{array}{l}\text { Minéraux }(g / l) \\
\text { Calcium } \\
\text { Sodium } \\
\text { Magnésium } \\
\text { Phosphore }\end{array}$ & $\begin{array}{l}1,13(0,13) \\
0,43(0,01) \\
0,12(0,02) \\
0,93(0,07)\end{array}$ & $\begin{array}{l}1,08(0,07) \\
0,43(0,02) \\
0,13(0,04) \\
0,90(0,05)\end{array}$ & $\begin{array}{l}1,14(0,11) \\
0,43(0,01) \\
0,13(0,03) \\
1,01(0,12)\end{array}$ & $\begin{array}{l}1,17(0,09) \\
0,45(0,03) \\
0,15(0,03) \\
0,93(0,02)\end{array}$ & $\begin{array}{l}1,13(0,10) \\
0,44(0,02) \\
0,13(0,03) \\
0,94(0,08)\end{array}$ \\
\hline $\begin{array}{l}\mathrm{pH} \\
\text { Taille micellaire }(\mathrm{nm})\end{array}$ & $\begin{array}{c}6,70(0,11) \\
193(6)\end{array}$ & $\begin{array}{c}6,74(0,08) \\
189(5)\end{array}$ & $\begin{array}{c}6,68(0,13) \\
197(4)\end{array}$ & $\begin{array}{c}6,68(0,09) \\
192(3)\end{array}$ & $\begin{array}{c}6,70(0,10) \\
193(5)\end{array}$ \\
\hline $\begin{array}{l}R \text { (min) } \\
K_{20}(\min )\end{array}$ & $\begin{array}{l}31,2(6,5) \\
49(15)\end{array}$ & $\begin{array}{l}28,7(8,2) \\
45(17)\end{array}$ & $\begin{array}{l}28,4(6,4) \\
35(13)\end{array}$ & $\begin{array}{l}27,5(5,8) \\
31(11)\end{array}$ & $\begin{array}{l}29,0(6,1) \\
40(15)\end{array}$ \\
\hline
\end{tabular}

Différences significative à ** 0,$05 ;{ }^{* * *} 0,01$.

Difference significant at **0.05; ${ }^{* *} 0.01$.

Les teneurs en azote et en MS du lactosérum augmentent (tableau II) mais cette augmentation n'est significative que pour la teneur en azote $(P<0,01)$.
Le rendement frais varie significativement avec le niveau de $\operatorname{TP}(P<0,05)$ sans qu'il y ait augmentation régulière : les laits correspondant au niveau de TP : 30,1 g/l 
Tableau II. Caractéristiques des transformations et des fromages fabriqués par niveau de TP. Characteristics of cheese making and cheeses by level of true protein content.

$$
\begin{aligned}
& \mathrm{TP}=29,4 \mathrm{~g} / \mathrm{TP}=30,1 \mathrm{~g} / \mathrm{TP}=30,8 \mathrm{~g} / \mathrm{TP}=32,2 \mathrm{~g} / \mathrm{Ensemble} \\
& \mathrm{n}=4 \quad \mathrm{n}=3 \quad \mathrm{n}=4 \quad \mathrm{n}=4 \quad \mathrm{n}=15
\end{aligned}
$$

\begin{tabular}{|c|c|c|c|c|c|}
\hline $\begin{array}{l}\text { Quantité lactosérum } 24 \mathrm{~h} \\
\text { (kg/100 kg de lait) }\end{array}$ & $83,32(0$ & $83,73(0,51)$ & $82,89(0,71)$ & $82,08(0,66)$ & $82,96(0,79)$ \\
\hline $\begin{array}{l}\text { Teneur en N lactosérum } \\
(\mathrm{g} /)^{* * *}\end{array}$ & ,39 $(0,04)$ & 1) & 1,51 & 4) & 7) \\
\hline rum (\%) & 3) & म) & 4) & 1) & 6 , \\
\hline $\begin{array}{l}\text { Rdt frais } J+1(\mathrm{~kg} / 100 \mathrm{~kg})^{* *} \\
\text { Extrait sec } J+1(\%) \\
\text { Rdt ajusté } 35,5 \% \mathrm{MS} \\
J_{+1}(\mathrm{~kg} / 100 \mathrm{~kg})^{* * *} \\
\text { Rdt sec } J_{+1}(\mathrm{~kg} / 100 \mathrm{~kg})^{* * *}\end{array}$ & $\begin{array}{l}15,11(0,41) \\
34,6(0,4) \\
14,59(0,31)\end{array}$ & $\begin{array}{l}14,88(0,50) \\
35,6(0,4) \\
14,95(0,35)\end{array}$ & $\begin{array}{l}15,36(0,43) \\
35,6(1,9) \\
15,39(0,76)\end{array}$ & $\begin{array}{l}15,95(0,38) \\
36,1(1,3) \\
16,31(0,48)\end{array}$ & $\begin{array}{l}15,36(0,55) \\
35,5(1,2) \\
15,35(0,81)\end{array}$ \\
\hline
\end{tabular}

Différence significative au seuil de ${ }^{* *} 0,05 ;{ }^{* * *} 0,01$.

Difference significant at ${ }^{* *} 0.05 ;{ }^{* *} 0.01$.

\% lactosérum / quantité d'eau du lait mise en service

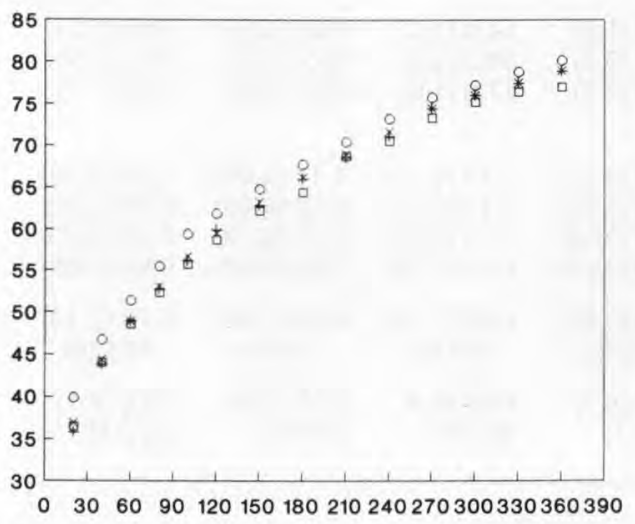

Temps d'égouttage ( $\mathrm{min}$ )

Fig 1. Cinétique d'égouttage des caillés. Niveau $T P: 000$ TP $=29,4 \mathrm{~g} / ; \mathrm{xxx}$ TP $=30,8 \mathrm{~g} / ;+++$ TP $=30,1 \mathrm{~g} / \mathrm{l} ; \quad T P=32,2 \mathrm{~g} / \mathrm{l}$.

Draining kinetics. True protein level content: $000 \mathrm{TP}=29,4 \mathrm{~g} / ; x x x \mathrm{TP}=30,8 \mathrm{~g} / ;+++\mathrm{TP}=$ $30,1 \mathrm{~g} / \mathrm{l}: \quad \mathrm{TP}=32,2 \mathrm{~g} / \mathrm{l}$. présentent en moyenne un rendement fromager frais plus faible que les laits à niveau de TP: 29,4 g/l (tableau II).

La teneur en MS des fromages a tendance à s'accroître avec le niveau de TP mais les différences ne sont pas significatives.

Avec la pris en compte des variations de teneur en MS des fromages, le rendement fromager ajusté à $35,5 \%$ de MS et le rendement fromager sec augmentent significativement avec le niveau de TP $(P<$ 0,01) (tableau II).

\section{Estimation des variations du rendement fromager à partir des variations des teneurs en protéines et du taux butyreux}

\section{Rendement frais}

La teneur en protéines ou la teneur en $M A C$ explique $68 \%$ des variations du ren- 
Tableau III. Équations de régression du rendement fromager établies à partir de la composition chimique du lait et des paramètres de la coagulation.

Regression equations of cheese yield based on milk chemical composition and coagulation parameters.

\begin{tabular}{|c|c|c|c|c|}
\hline $\begin{array}{l}\text { Variable } \\
\text { expliquée }\end{array}$ & $\begin{array}{l}\text { Variables explicatives } \\
\text { au seuil de } 5 \% \\
\text { (Teneurs exprimées } \\
\text { en g/l de lait) }\end{array}$ & Équation de régression & $\begin{array}{l}\text { Coefficient } \\
\text { détermination } \mathrm{r}^{2} \\
(\%)\end{array}$ & $\begin{array}{l}\text { Écart type } \\
\text { résiduel }\end{array}$ \\
\hline \multirow[t]{2}{*}{$\begin{array}{l}\text { Rdt frais } J_{+1} \\
\text { (kg/100 kg lait) }\end{array}$} & $\begin{array}{l}\text { TP TB } \\
\text { MAC TB } \\
\text { TCas TB } \\
\beta-C N\end{array}$ & $\begin{array}{l}\operatorname{Rdt}_{\text {frais } J+1}=0,37+0,36 T P+0,15 T B \\
\operatorname{Rdt}_{\text {frais } J+1}=-0,60+0,46 \mathrm{MAC}+0,18 \mathrm{~TB} \\
\operatorname{Rdt}_{\text {frais } J+1}=0,18+0,37 \mathrm{TCas}+0,21 \mathrm{~TB} \\
\mathrm{Rdt}_{\text {frais } J+1}=8,59+0,007 \beta-\mathrm{CN}\end{array}$ & $\begin{array}{l}76,5 \\
80,6 \\
59,7 \\
63,3\end{array}$ & $\begin{array}{l}0,29 \\
0,26 \\
0,38 \\
0,35\end{array}$ \\
\hline & $\begin{array}{l}K_{20} \\
K_{20} T P\end{array}$ & $\begin{array}{l}\operatorname{Rdt}_{\text {frais } J+1}=16,66-0,033 K_{20} \\
\operatorname{Rdt}_{\text {frais } J+1}=9,75-0,021 K_{20}+0,211 T P\end{array}$ & $\begin{array}{l}73,9 \\
85,6\end{array}$ & $\begin{array}{l}0,29 \\
0,23\end{array}$ \\
\hline $\begin{array}{l}\text { Rdt ajusté en MS J+1 } \\
\text { (kg/100 kg lait) }\end{array}$ & $\begin{array}{l}\text { TP } \\
\text { MAC } \\
T C \text { Cas } \\
\alpha_{\mathrm{s}}-\mathrm{CN} \beta-\mathrm{CN} \gamma \kappa-\mathrm{CN}\end{array}$ & 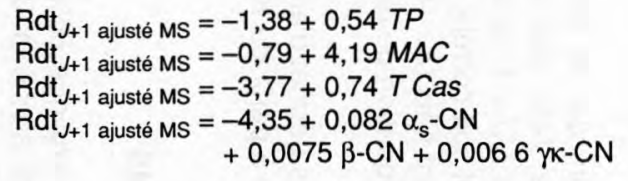 & $\begin{array}{l}65,6 \\
61,4 \\
81,0 \\
81,2\end{array}$ & $\begin{array}{l}0,49 \\
0,52 \\
0,36 \\
0,39\end{array}$ \\
\hline $\begin{array}{l}\text { Rdt sec } J_{+1} \\
\text { (kg MS/100 kg lait) }\end{array}$ & $\begin{array}{l}\text { TP } \\
\text { MAC } \\
T C \text { Cas } \\
\beta-C N \alpha_{s}-C N \gamma \kappa-C N\end{array}$ & $\begin{aligned} \operatorname{Rdt}_{\sec J+1} & =-0,07+0,18 \mathrm{TP} \\
\operatorname{Rdt}_{\sec J+1} & =0,10+0,22 \mathrm{MAC} \\
\operatorname{Rdt}_{\sec J+1}= & -0,62+0,23 \mathrm{TCas} \\
\operatorname{Rdt}_{\sec J+1}= & -0,85+0,0026\left(\beta-\mathrm{CN}+\alpha_{\mathrm{s}}-\mathrm{CN}\right) \\
& +0,0019 \gamma \mathrm{k}-\mathrm{CN}\end{aligned}$ & $\begin{array}{l}71,5 \\
67,4 \\
81,7 \\
83,1\end{array}$ & $\begin{array}{l}0,14 \\
0,15 \\
0,11 \\
0,12\end{array}$ \\
\hline
\end{tabular}


dement. La prédiction est améliorée de 9$12 \%$ avec la prise en compte du TB (tableau III).

La teneur en caséines totales n'explique que $41 \%$ des variations du rendement, le cumul des variables taux de caséines et $T B, 60 \%$. Parmi les différentes caséines du lait, seule la teneur en caséines $\beta(\beta-C N)$ du lait $(g / l)$ est significativement liée au rendement frais $(P<0,05)$, le $T B$ n'intervenant plus significativement (tableau III).

Le temps d'obtention de la fermeté standard $\left(K_{20}\right)$ explique $74 \%$ des variations du rendement frais à $J+1$, le temps de coagulation, bien qu'il soit corrélé significativement au rendement frais $(r=-0,52)$, n'est pas retenu dans l'équation de régression (seuil de $5 \%$ ).

La teneur en protéines permet d'améliorer la prédiction obtenue à partir du $K_{20}$ de $12 \%$ (tableau III).

\section{Rendement ajusté en MS}

La teneur en protéines explique $66 \%$ des variations du rendement ajusté à $35,5 \%$ de MS, la teneur en MAC $61 \%$ et la teneur en caséines $81 \%$ (tableau III). Les teneurs (g/l) des 3 grands types de caséines sont corrélées significativement au rendement ajusté en MS, leur cumul explique la même part de variation que la teneur en caséines totales.

Après la prise en compte des teneurs en différentes protéines, le TB n'a plus d'effet significatif sur le rendement. Le temps de coagulation et le $K_{20}$ ne sont pas des paramètres explicatifs au seuil de $5 \%$.

\section{Rendement sec}

Les niveaux de prédiction du rendement sec sont respectivement de $71 \%, 67 \%$ et
$82 \%$ pour la teneur en protéines, la teneur en $M A C$ et la teneur en caséines totales, le $T B$ n'ayant alors plus d'effet significatif sur le rendement.

Ni le temps de coagulation ni le $K_{20}$ ne sont explicatifs des variations du rendement sec au seuil de $5 \%$.

\section{DISCUSSION}

\section{Caractéristiques des fromages fabriqués}

La teneur en MS des fromages mesurée au démoulage tend à augmenter avec le niveau de TP mais ces variations ne sont pas significatives. Si l'effet négatif de la teneur en matières grasses sur la synérèse est bien établi (Marshall, 1982; Storry et al, 1983; Weber, 1984; Grandison et al, 1984, 1985), l'influence des protéines ou des caséines a été moins étudiée : Marshall (1982) et Storry et al (1983) notent un ralentissement de la synérèse, mesurée jusqu'à $70 \mathrm{~min}$ après tranchage, avec l'augmentation de la teneur en composants colloïdaux ou en caséines. Gilles et Lawrence (1985) ne mesurent pas de variation du pourcentage d'humidité des fromages suite à l'augmentation du taux de protéines du lait.

Dans cet essai, l'augmentation de la teneur en MS des fromages à $J+1$ pour les fromages à TP élevé est associée à une expulsion de lactosérum (exprimée en pourcentage par rapport à l'eau du lait mis en œuvre) plus faible en début d'égouttage. Le TB étant peu variable d'un groupe à l'autre, cette diminution de la synérèse de début d'égouttage correspondrait à l'augmentation de la teneur en caséines pour les laits à TP élevé. 


\section{Estimation des variations du rendement à partir des variations des paramètres de composition chimique}

\section{Rendement frais}

Le coefficient de détermination du rendement frais à partir de la teneur en protéines et du taux butyreux est élevé $(76 \%)$ mais l'incertitude découlant des pertes entre le poids de lait mis en œuvre d'une part, et les poids de caillé et de lactosérum d'autre part $(-1,7 \% \pm 0,6 \%)$ conduit à considérer avec prudence les coefficients de régression attribués à chaque composant.

La teneur en caséines n'améliore pas la prédiction par rapport à celle établie à partir de la teneur en protéines. De plus, après prise en compte de la teneur en protéines, la proportion de caséines dans les protéines n'est plus retenue dans la régression (seuil de 5\%). Maubois et al (1970) notaient une légère amélioration du coefficient de détermination avec l'utilisation du taux de caséine au lieu du taux de protéine $(+5-6 \%)$ ou du cumul taux de protéine et part des caséines dans les protéines par rapport aux taux de protéine seul $(+6-14 \%)$. Dans cet essai, la proportion de caséines dans les protéines totales n'a pas varié significativement et le TP suffit à estimer l'aptitude fromagère des laits.

Le modèle établi à partir de la teneur en $M A C$ et du $T B$ est légèrement meilleur que celui établi à partir de la teneur en protéines et du TB. Mais la détermination de la teneur en $M A C$ nécessite une coagulation du lait par la présure.

Le rôle important joué par la $\beta-C N$ est montré par le bon niveau de prédiction du rendement frais à partir de sa teneur. Le pourcentage de $\beta$-CN est corrélé significativement au rendement frais $(r=+0,56)$ alors que ceux des autres caséines ne le sont pas. Ces résultats confortent ceux obtenus par Ali et al (1980), Marziali et $\mathrm{Ng}$ Kwai Hang (1986) et Cossin (1988) en fabrication de pâte pressée.

Cette influence de la caséine $\beta$ sur le rendement fromager frais peut provenir, d'une part, de son éventuelle relation positive avec la quantité de matière utile du lait, d'autre part d'un éventuel effet sur la rétention en eau du fromage. Parmi les constituants du lait, la proportion de $\beta-C N$ n'est corrélée significativement qu'au TB. Sa corrélation positive avec le rendement frais peut provenir en partie de cette augmentation du TB pour les laits les plus riches en $\beta-C N$. Par ailleurs, les travaux réalisés par Pearse et al (1986), Pearse et MacKinlay (1989) et Hardy (1990) montrent que la $\beta-\mathrm{CN}$ intervient surtout au niveau de l'égouttage en favorisant celui-ci : elle interviendrait par le biais de ses groupements phosphorylés chargés négativement qui détermineraient l'orientation de la $\beta-C N$ à la surface de la micelle et permettraient des interactions ioniques intermicellaires, favorisant ainsi la contraction du coagulum et l'expulsion du sérum (Pearse et MacKinlay, 1989). Pourtant, dans cet essai, la proportion de lactosérum égoutté aux différents temps n'a pas varié significativement avec la concentration en $\mathrm{B}-\mathrm{CN}$.

Les résultats de la bibliographie concernant le rôle favorable de la $\beta$-CN sur la synérèse et la corrélation positive établie dans cette étude entre sa teneur ou son pourcentage et le rendement frais peuvent sembler contradictoires. En fait, l'influence favorable de la caséine $\beta$ sur l'expulsion de lactosérum en début d'égouttage et donc sur la vitesse d'égouttage d'une part, son effet sur le volume de lactosérum expulsé en fin d'égouttage et donc sur la rétention en eau du fromage d'autre part, constituent sans doute 2 phénomènes indépendants. 


\section{Rendement ajusté en MS et rendement sec}

Les variations de la teneur en matière grasse du lait (entre 24,5 et $28,8 \mathrm{~g} / \mathrm{l}$ ) n'interviennent pas significativement dans la détermination ni du rendement ajusté en MS, ni du rendement sec. Pourtant le TB des laits, même après écrémage partiel, présente une variabilité aussi importante que la teneur en protéines ( $C V=4 \%)$.

Les niveaux de prédiction à partir de la composition chimique sont légèrement inférieurs pour le rendement ajusté en MS à ceux du rendement sec. Ces coefficients de régression attribués aux différents composants doivent être considérés avec prudence compte tenu de l'incertitude découlant des pertes entre la matière sèche du lait mise en œuvre et la quantité de matière sèche du caillé et du lactosérum $(-2,9 \% \pm 2,8 \%)$.

La teneur du lait en caséines totales améliore les prédictions par rapport à sa teneur en protéines. La teneur en caséines traduirait mieux la quantité de matière sèche retenue, alors que la teneur en protéines totales reflèterait mieux la rétention en eau.

Les coefficients de détermination pour les équations établies à partir de la teneur en caséines ou du cumul teneur en protéines et proportion de caséines dans les protéines sont les mêmes. Maubois et al (1970) ont observé une amélioration de la prédiction avec la prise en compte de la part des caséines dans les protéines mais pas avec l'utilisation de la teneur en caséines au lieu de la teneur en protéines.

La prépondérance de la $\beta$-CN n'est pas retrouvée en ce qui concerne l'explication du rendement sec. Parmi les 2 voies possibles quant à son influence sur le rendement frais, ce résultat conforte l'hypothèse émise quant à l'influence de la $\beta-\mathrm{CN}$ sur la rétention en eau de fromage.

\section{Estimation des variations du rendement à partir des variations des paramètres technologiques et de composition chimique}

\section{Rendement frais}

Le temps d'obtention de la fermeté standard est bien lié au rendement fromager frais $(r=-0,86)$ (tableau IV). Le temps de coagulation, bien que corrélé au rendement frais $(r=-0,52)$, n'est pas retenu dans le modèle après prise en compte du $K_{20}$. Dans ces essais, les transformations ne sont pas effectuées à technologie constante car les variations du temps de coagulation déterminent le rythme des différentes étapes de la transformation (tranchage, moulage).

Avec des mesures au Formagraph, Aleandri et al (1989) et Hurtaud et al (1991) obtiennent les meilleurs coefficients de détermination des variations du rendement fromager brut avec la fermeté du caillé mesurée 30 min après emprésurage $\left(A_{30}\right)$, mais Hurtaud et al (1991) obtiennent également des coefficients élevés avec $K_{20}$ et $R$, pour des rendements mesurés après centrifugation d'un lait emprésuré.

Le temps d'obtention de la fermeté standard est par ailleurs corrélé négativement à la teneur en protéines et à la teneur en MAC (tableau IV), ce qui correspond aux résultats de la littérature, mais la corrélation positive avec la proportion de caséines dans les protéines et l'absence de corrélation avec la teneur en caséines sont plus surprenantes.

La prédiction établie à partir des composantes chimiques (TP $+T B$ ) est meilleure que celle établie à partir des paramètres de la coagulation seuls, confirmant ainsi les observations effectuées par Aleandri et al (1989) en fabrication de Parmesan.

L'utilisation simultanée du $K_{20}$ et de la teneur en protéines améliore la prédiction 
Tableau IV. Matrice de corrélations entre les variables de composition chimique, les paramètres de la coagulation et les rendements fromagers à $J$ +1 . Seules les corrélations significatives au seuil de $5 \%$ sont représentées.

Correlations between chimical composition characteristics, coagulation parameters and cheese yield at day +1 . Only significance levels at $5 \%$ have been shown.

\begin{tabular}{|c|c|c|c|c|c|c|c|c|c|c|c|c|c|c|c|}
\hline & $\begin{array}{l}\text { Extrait } \\
\text { sec }\end{array}$ & TB & TP & T Cas & $\begin{array}{l}\text { Cas/Prot } \\
\text { (\%) }\end{array}$ & MAC & $\begin{array}{l}\alpha_{s}-C N \\
(\%)\end{array}$ & $\begin{array}{l}\beta-C N \\
(\%)\end{array}$ & $\begin{array}{l}\gamma \kappa-C N \\
(\%)\end{array}$ & R & $\mathrm{K}_{20}$ & $\begin{array}{l}R d t \\
\text { frais }\end{array}$ & ESJ1 & $\begin{array}{l}\text { Rdt } \\
\text { ajusté } \\
\text { MS }\end{array}$ & $\begin{array}{l}R d t \\
\text { sec }\end{array}$ \\
\hline$T B$ & & & & & & & & & & & & & & & \\
\hline $\begin{array}{l}T P \\
T \text { Cas } \\
\text { Cas/Prot (\%) }\end{array}$ & $+0,50$ & & $+0,90$ & & & & & & & & & & & & \\
\hline $\begin{array}{l}M A C \\
\alpha_{s}-C N \quad(\%)\end{array}$ & $+0,53$ & & $+0,98$ & $+0,92$ & & & & & & & & & & & \\
\hline $\begin{array}{l}\beta-\mathrm{CN} \quad(\%) \\
\gamma \kappa-\mathrm{CN} \\
R\end{array}$ & & $\begin{array}{l}+0,65 \\
-0,61\end{array}$ & & & $\begin{array}{l}+0,57 \\
+0,55\end{array}$ & & & & & & & & & & \\
\hline$K_{20}$ & $-0,55$ & & $-0,66$ & & $+0,68$ & $-0,63$ & & & & $+0,72$ & & & & & \\
\hline $\begin{array}{l}\text { Rdt frais } J 1 \\
\text { ES } J 1\end{array}$ & $+0,77$ & & $+0,82$ & $\begin{array}{l}+0,64 \\
+0,55\end{array}$ & & $+0,83$ & & $+0,56$ & & $\begin{array}{l}-0,52 \\
+0,50\end{array}$ & $-0,86$ & & & & \\
\hline $\begin{array}{l}\text { Rdt ajusté MSJ1 } \\
\text { Rdt } \sec J 1\end{array}$ & & & $\begin{array}{l}+0,81 \\
+0,84\end{array}$ & $\begin{array}{l}+0,80 \\
+0,90\end{array}$ & & $\begin{array}{l}+0,78 \\
+0,82\end{array}$ & & & & & & $\begin{array}{l}+0,60 \\
+0,67\end{array}$ & $\begin{array}{l}+0,72 \\
+0,65\end{array}$ & $+0,99$ & \\
\hline
\end{tabular}


établie à partir de la composition chimique seule $(+9 \%$ ) (tableau III).

\section{CONCLUSION}

Pour des transformations en pâtes molles effectuées sur des laits de mélange de plusieurs troupeaux, les teneurs en protéines et en matière grasse permettent d'expliquer $77 \%$ des variations du rendement fromager frais. Après prise en compte des différences de teneur en MS des fromages, seul le TP explique une part des variations : $66 \%$ pour le rendement ajusté en MS et $71 \%$ pour le rendement sec.

La prédominance de la teneur en caséines totales par rapport à la teneur en protéines totales pour expliquer les variations de rendement n'apparaît que pour le rendement ajusté en MS ou le rendement sec. La proportion de caséines totales dans les protéines totales a peu varié, la prise en compte de ce paramètre est sans doute nécessaire pour des laits dont le nombre caséique varie plus fortement (laits de fin de lactation, de mammite, etc).

Par contre, la caséine $\beta$ semble jouer un rôle important en transformation fromagère. En effet, la corrélation positive établie non seulement avec la teneur en $\beta$-CN mais avec sa proportion dans les caséines totales et le rendement fromager frais d'une part, l'absence de corrélation significative entre la proportion de $\beta-C N$ et le rendement fromager ajusté en MS ou le rendement fromager $\mathrm{sec}$ d'autre part, conduit à émettre l'hypothèse d'une influence de la $\beta-C N$ sur la rétention en eau du fromage. Afin de conforter cette hypothèse, il serait intéressant de transformer des laits à proportion de $\beta-C N$ variable et de mesurer la cinétique d'égouttage de ces laits, le rendement fromager et la rétention en eau des fromages obtenus. $\mathrm{Ce}$ type d'essais permettrait également de préciser les rôles pouvant paraître antago- nistes de la $\beta-C N$, d'une part sur l'expulsion du lactosérum, et d'autre part sur la rétention en eau du fromage.

Après prise en compte de la teneur en $\beta$ - $C N$, le TB n'intervient plus pour expliquer les variations du rendement frais. Les prédictions établies à partir du cumul (TP + $T B$ ) ou ( $T$ Cas + TB) sont meilleures que celle établie à partir de la teneur en $\beta-C N$.

Le temps d'obtention de la fermeté standard est un bon prédicteur du rendement frais, mais il ne permet pas d'expliquer une part aussi importante des variations du rendement que le cumul $(T P+T B)$. II permet cependant de rendre compte d'une partie des phénomènes complexes intervenant lors de la transformation du lait en caillé en présentant l'avantage par rapport aux paramètres chimiques d'une vision plus globale. La meilleure prédiction du rendement frais est obtenue par l'utilisation simultanée de la composition chimique du lait et des paramètres de la coagulation. Malgré ces résultats pour le rendement frais, l'absence de corrélation entre les seuls paramètres de la coagulation et le rendement fromager exprimé en MS ou corrigé de la teneur en MS laisse ouverte l'interrogation concernant l'utilisation des seuls paramètres de la coagulation pour caractériser l'aptitude fromagère des laits.

\section{REMERCIEMENTS}

Cet essai s'inscrit dans un programme de recherche ayant bénéficié du soutien financier de l'Association nationale de la recherche technique, de la Direction générale de l'alimentation, des industriels laitiers réunis au sein d'Arilest et de la Région Lorraine.

\section{RÉFÉRENCES}

Aleandri R, Schneider JC, Butazzoni LG (1989) Evaluation of milk cheese production based 
on milk characteristics and formagraph measures. J Dairy Sci 72, 1967-1975

Ali AE, Andrews AT, Cheeseman GC (1980) Factors influencing casein distribution in cold-stored milk and their effects on cheesemaking parameters. J Dairy Res 47, 383391

Andrews AT, Taylor MD, Owen AJ (1985) Rapid analysis of bovine milk proteins by fast protein liquid chromatography. $J$ Chromatogr 348, 177-185

Banks JM, Muir DD, Tamine AY (1984) Equations for estimation of the efficiency of Cheddar cheese production. Dairy Ind Int 49 (4), 14-17

Cossin V (1988) Contribution à l'étude des aptitudes fromagères du lait de vache. Thèse Docteur Ingénieur, INA Paris Grignon

Emmons DB, Ernstrom CA, Lacroix C, Verret $P$ (1990) Predictive formulas for yield of cheese from composition of milk: a review. J Dairy Sci 73, 1365-1394

Garel JP, Coulon JB (1990) Effet de l'alimentation et de la race des vaches sur la fabrication de fromage d'Auvergne de SaintNectaire. INRA Prod Anim 3, 127-136

Gilles J, Lawrence RC (1985) The yield of cheese. N Z J Dairy Sci Technol 20, 205214

Grandison AS, Ford GD, Millard D, Owen AJ (1984) Chemical composition and coagulating properties of renneted milks from cows during early lactation. J Dairy Res 51, 407416

Grandison AS, Ford GD, Millard D, Anderson $M$ (1985) Interrelationships of chemical composition and coagulation properties of renneted milks from dairy cows grazing ryegrass or white clover. J Dairy Res 52, 41-46

Hardy C (1990) Variabilité de la composition protéique des laits : origines zootechniques et conséquences technologiques en pâte pressée cuite. Thèse Docteur Ingénieur, Rennes

Hurtaud C, Vérité R, Rulquin H (1991) Dètermination de l'aptitude des laits à la transformation fromagère : intérêt et limites des tests de laboratoire. Journées sur la qualité des laits à la production et aptitude fromagère. Colloque INRA-ENSA Rennes, 23-24 janvier

Kerjean JR (1984) Conséquences fromagères des variations de composition du lait. Qualité chimique du lait de fromagerie. In: La composition chimique du lait et ses incidences technologiques. Journées INRA ENSAR INAPG, septembre

Marshall RJ (1982) An improved method for measurement of the syneresis of curd formed by rennet action on milk. $J$ Dairy Res 49, 329-336

Marziali AS, Ng Kwai Hang KF (1986) Relationships between milk protein polymorphisms and cheese yielding capacity. J Dairy Sci 69, 1193-1201

Maubois JL, Mocquot G (1967) Comment ramener à la même teneur en substance sèche des fabrications de fromage en vue de comparer les "rendements" respectifs du lait en fromage. Rev Lait Fr 239, 15-18

Maubois JL, Ricordeau G, Mocquot G (1970) Étude des rendements en fromagerie de $\mathrm{Ca}$ membert et de Saint-Paulin. Lait 50, 351 . 373

Mocquot G, Ricordeau G, Auriol P (1963) Estimation du rendement en fromage gruyère de Comté en fonction de la richesse du lait de chaudière. Ann Zootech 12, 53-66

Pearse MJ, Linklater PM, Hall RJ, MacKinlay AG (1986) Effect of casein micelle composition and casein dephosphorylation on coagulation and syneresis. J Dairy Res 53,381 390

Pearse MJ, MacKinlay AG (1989) Biochemical aspects of syneresis: a review. J Dairy $\mathrm{Sci}$ $72,1401-1407$

Ribadeau-Dumas B, Grappin R (1989) Milk protein analysis. Lait 69, 357-416

Storry JE, Grandison AS, Millard D, Owen AJ, Ford GD (1983) Chemical composition and coagulating properties of renneted milks from different breeds and species of ruminants. J Dairy Res 50, 215-229

Weber F (1984) L'égouttage du coagulum. In: Le fromage (Eck A, ed) Lavoisier-Tech et Doc, Paris, 22-36 